\title{
Coronary Sinus Thrombosis in a Patient Without History of Endothelial Cardiac Injury
}

\author{
Anton Mararenko ${ }^{\mathrm{a}, \mathrm{c}}$, Abbas Alshami $^{\mathrm{a}}$, Firas Ajam ${ }^{\mathrm{b}}$, David Zagha ${ }^{\mathrm{b}}$
}

\begin{abstract}
Coronary sinus thrombosis (CST) is a rare but life-threatening condition that involves clot formation within the vessel responsible for draining all of the venous blood from the myocardium itself. The coronary sinus is situated in the right atrium approximately half-way between the tricuspid value and the inferior vena cava. The coronary sinus is rarely cited in medical literature due to limited knowledge as well as rarity in clinical encounters. CST can be a rapidly progressive life-threatening emergency as the interruption of vascular drainage can result in pericardial effusions, tamponade and cardiogenic shock. A major clinical challenge in diagnosing and treating this condition is due to relative rarity as well as the non-specificity of presenting symptoms that are often associated with more commonly encountered cardiopulmonary diseases. CST is most commonly induced by endothelial damage, such as post intracardiac instrumentation with catheter guidewires, or any of the criteria outlined by Virchow's triad. Our team described the finding of a thrombus $1.8 \mathrm{~cm}$ in diameter in a patient with underlying hepatobiliary cancer as well as underlying bacteremia from infected ascitic fluid. Though our patient remained hemodynamically stable without cardiopulmonary complications, we hope to spark a discussion within the medical community to increase awareness as well as to highlight the need for more research on this potentially life-threatening condition.
\end{abstract}

Keywords: Coronary sinus; Thrombosis; Bacteremia; Anticoagulation; Echocardiogram

\section{Introduction}

Coronary sinus thrombosis (CST) is an incredibly rare condition characterized by thrombus formation in the vessel responsi-

Manuscript submitted May 24, 2021, accepted June 22, 2021

Published online August 25, 2021

${ }^{a}$ Department of Internal Medicine, Jersey Shore University Medical Center, Neptune, NJ, USA

bDepartment of Cardiology, Jersey Shore University Medical Center, Neptune, NJ, USA

${ }^{\mathrm{c} C o r r e s p o n d i n g ~ A u t h o r: ~ A n t o n ~ M a r a r e n k o, ~ D e p a r t m e n t ~ o f ~ I n t e r n a l ~ M e d i c i n e, ~}$ Jersey Shore University Medical Center, 1945 Route 33, Neptune, NJ 07753, USA. Email: Anton.Mararenko@hmhn.org

doi: https://doi.org/10.14740/jmc3726 ble for draining venous blood from the cardiac veins. The most common findings that are suggestive of CST include shortness of breath, chest pain or any ischemic findings on electrocardiogram $[1,2]$. Unfortunately, diagnosis of CST remains a medical challenge as the previously mentioned findings are often associated with the more commonly encountered cardiopulmonary diseases, such as acute coronary syndrome, heart failure, pneumonia and chronic obstructive pulmonary disease. If present, early diagnosis is critical as patients have been found to have rapid deterioration with a high mortality rate if untreated [3]. When suspected, CST can be appreciated on echocardiogram or computed tomography imaging with vascular contrast [4]. Most published cases of CST have been found in patients that have underwent recent cardiac instrumentation or are known to have a prothrombotic state, as summarized by Virchow's triad, that naturally increase the clot burden. Despite the high mortality of the condition, there is very limited research on the management of this life-threatening condition. This is partially due to diagnosis on autopsy as awareness and clinical suspicion of this rare condition remain low.

Our team presented a patient that was incidentally found to have a CST while being worked up for worsening, decompensated heart failure. Our case stands in stark contrast to previously reported incidences as our patient did not undergo any cardiac instrumentation and had no history of coronary artery disease. Notably, our patient was found to have presumed pancreatic cancer with metastasis to the liver.

\section{Case Report}

Our team described a 64-year-old female with a past medical history significant for a remote history of recurrent lymphangiomatosis, recently diagnosed cervical adenocarcinoma, and presumed metastatic pancreatic cancer that presented with worsening weakness and significant gastrointestinal distress. In the past, the patient underwent repeated drainage of effusions secondary to lymphangiomatosis. The lymphangiomatosis continued to worsen and resulted in pulmonary and cardiac involvement leading to cardiac tamponade requiring a pericardial window in 2004. Ultimately, the patient underwent video-assisted thorascopic surgery and partial thoracic duct embolization. Prior to the described admission, the patient had a recent admission for abdominal discomfort and was found to have a non-bleeding gastric ulcer. The biopsy report was negative for signs of malignancy; however, she did have a 
Table 1. Laboratory Results

\begin{tabular}{lll}
\hline Laboratory test & Value & Reference range \\
\hline White blood cell count & $13.3 \times 10^{3} / \mu \mathrm{L}$ & $4.5-11 \times 10^{3} / \mu \mathrm{L}$ \\
Hemoglobin & $5.6 \mathrm{~g} / \mathrm{dL}$ & $12-16 \mathrm{~g} / \mathrm{dL}$ \\
MCV & $92.9 \mathrm{fL}$ & $80-100 \mathrm{fL}$ \\
Platelet count & $109 \times 10^{3} / \mu \mathrm{L}$ & $140-450 \times 10^{3} / \mu \mathrm{L}$ \\
Creatinine & $2.70 \mathrm{mg} / \mathrm{dL}$ & $0.44-1.00 \mathrm{mg} / \mathrm{dL}$ \\
AST & $63 \mathrm{U} / \mathrm{L}$ & $10-42 \mathrm{U} / \mathrm{L}$ \\
ALT & $46 \mathrm{U} / \mathrm{L}$ & $10-60 \mathrm{U} / \mathrm{L}$ \\
Total bilirubin & $18.1 \mathrm{mg} / \mathrm{dL}$ & $0.2-1.3 \mathrm{mg} / \mathrm{dL}$ \\
Anion gap & $16 \mathrm{mmol} / \mathrm{L}$ & $5-13 \mathrm{mmol} / \mathrm{L}$ \\
Bicarbonate & $20 \mathrm{mmol} / \mathrm{L}$ & $24-31 \mathrm{mmol} / \mathrm{L}$ \\
Alkaline phosphatase & $956 \mathrm{U} / \mathrm{L}$ & $38-126 \mathrm{U} / \mathrm{L}$ \\
Albumin & $1.1 \mathrm{~g} / \mathrm{dL}$ & $3.5-5.0 \mathrm{~g} / \mathrm{dL}$ \\
CEA & $100 \mathrm{ng} / \mathrm{mL}$ & $\mathrm{Undetermined}$ \\
CA19-9 & $56,560 \mathrm{U} / \mathrm{mL}$ & $0-35 \mathrm{U} / \mathrm{mL}$ \\
CA125 & $166.7 \mathrm{U} / \mathrm{mL}$ & $0-35 \mathrm{U} / \mathrm{mL}$ \\
Lactic acid & $1.9 \mathrm{mmol} / \mathrm{L}$ & $0.5-2.0 \mathrm{mmol} / \mathrm{L}$ \\
\hline
\end{tabular}

MCV: mean corpuscular volume; AST: aspartate transaminase; ALT: alanine transaminase; CEA: carcinoembryonic antigen; CA19-9: carbohydrate 19-9; CA125: cancer antigen 125.

progressively growing pancreatic mass resulting in obstructive jaundice. At the time the patient was treated with pancreatic duct dilation and stent placement. A pancreatic biopsy was not done at that time due to an unacceptably high risk of bleeding given the pattern of vascularity. Additionally, the patient was diagnosed with cervical adenocarcinoma shortly prior to the current admission. She did not receive any treatment prior to being hospitalized.

At the time of this admission, the patient presented with diffuse weakness, nausea associated with non-bloody and nonbilious vomiting, worsening swelling of the upper and lower extremities for 2 months and a decrease in appetite. Vital signs on admission were a heart rate of 90 beats per minute, respiratory rate of 18 breaths per minute, temperature of 98 ${ }^{\circ} \mathrm{F}$ measured orally and oxygen saturation of $98 \%$ on room air. Physical examination was significant for scleral icterus, abdominal distension with a fluid wave, extensive edema in the upper and lower extremities as well as diffuse jaundice. Cardiopulmonary examination did not reveal any acute findings. Of note, there was a chronic sacral lesion with peripheral erythema and edema. Laboratory workup including complete metabolic panel (CMP), complete blood counts (CBCs), and additional testing, as summarized in Table 1, were significant for a mild leukocytosis, severe anemia and acute kidney injury. Additionally, the creatinine on admission was $2.70 \mathrm{mg} / \mathrm{dL}$ but was as low as $0.68 \mathrm{mg} / \mathrm{dL}$ within the past year. There was also an elevated anion gap metabolic acidosis but a lactic acid that was within the normal range. The extent of biliary disease was also evident by the hyperbilirubinemia $(18.1 \mathrm{mg} / \mathrm{dL})$ and hypoalbuminemia $(1.1 \mathrm{~g} / \mathrm{dL})$.

Given the underlying abdominal distension, a computed tomography (CT) scan of the abdomen was significant for a 72 $\mathrm{mm}$ pelvic mass in addition to diffuse anasarca, severe intrahepatic and extrahepatic biliary duct dilatation with pneumobilia and a previously placed stent. Reflex CA19 and CA125 levels were significantly elevated to 56,560 and $166.7 \mathrm{U} / \mathrm{mL}$, respectively. Carcinoembryonic antigen (CEA) levels were non-elevated. Given the findings on CT imaging and the tumor markers, it is likely that the patient had a primary pancreatic cancer with metastasis to the liver.

Given the underlying abdominal findings on imaging, the patient underwent further intervention by gastroenterology and surgery. In order to rule out cardiac causes of peripheral edema, a transthoracic echocardiogram was performed. Imaging results were significant for an ejection fraction of $65-70 \%$ with no signs of diastolic dysfunction. Valvular evaluation was significant for moderate tricuspid regurgitation. Severe pulmonary hypertension was present as well. Most concerning, as seen in Figure 1, there was coronary sinus dilation with overlying thrombus present.

The patient underwent evaluation by multiple specialties given the extensive number of active medical problems on admission. The patient was treated with serial transfusions for the severe anemia. The leukocytosis was approached with initial administration of broad-spectrum antibiotics followed by serial blood cultures and body fluid cultures. Patient underwent a paracentesis due to severe ascites and cultures resulted positive for Klebsiella pneumonia. Blood cultures were also positive for Klebsiella pneumonia in two vials, each of which was drawn from a different site. Initially, patient was treated with broad spectrum antibiotics with repeat sets of blood cultures to monitor for resolution. Once the bacteremia had cleared and cultures had speciated, the patient completed a 2-week course of piperacillin-tazobactam $3.0-0.375 \mathrm{~g}$.

Unfortunately, after an extensive discussion with the patient and their next of kin, the patient had decided to abandon all aggressive measures and proceed with comfort care. The nature of the disease as well as diagnosis of CST had been thoroughly explained yet they refused to commit to anticoagulation or invasive intervention.

\section{Discussion}

The coronary sinus is a conglomerate of veins that drain the blood carried by the small, middle, great, posterior and oblique cardiac veins from the myocardium into the right atrium. It drains into an opening that is located approximately between the inferior vena cava and the tricuspid value. The coronary sinus receives minimal clinical attention as it is not well studied and associated with few pathologic states. However, when the coronary sinus is involved, the disease course can be rapidly progressive with a very high rate of mortality [3].

Several cases of coronary sinus have been reported in a multitude of conditions. The underlying pathophysiology of CST can be described by any state that induces endothelial damage or can induce a prothrombotic state. A healthy and intact endothelium helps to maintain equilibrium of hemostasis by providing an anticoagulant and antiplatelet surface. Many of these molecules are stored in the Weibel-Palade bodies. Thrombo- 


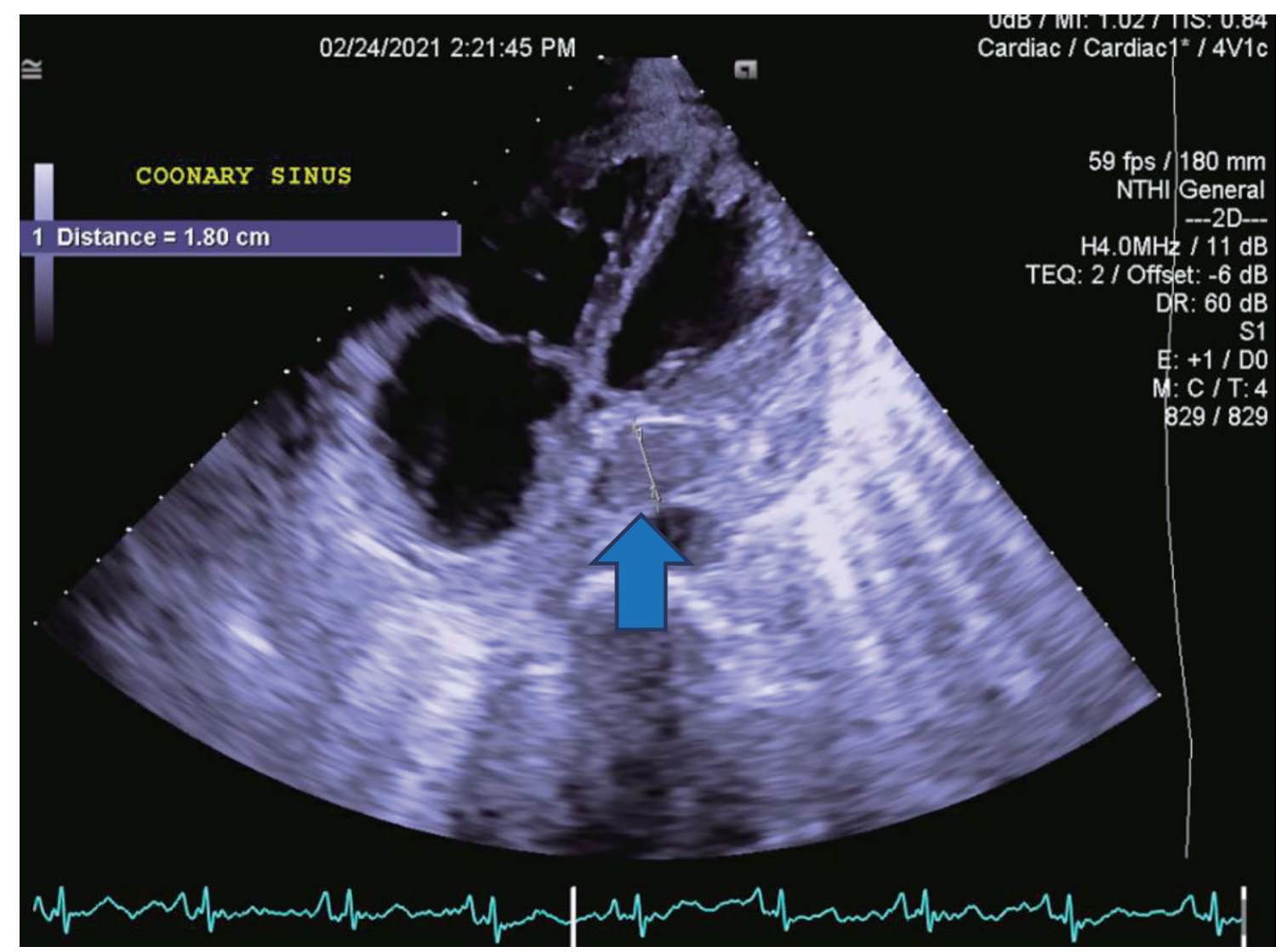

Figure 1. A transthoracic, long axis view on two-dimensional echocardiogram demonstrating coronary sinus thrombosis. The blue arrow represents the coronary sinus thrombosis that measures $1.8 \mathrm{~cm}$.

modulin, tissue factor and ectonucleotidases are a few examples of the glycoproteins that must be carefully regulated to prevent unintentional thrombosis [5]. Similarly, the endothelium is also responsible for the release of von Willebrand factor, a protein that induces thrombosis by cross linking platelets [6]. Most importantly, venous thrombosis is more likely by any condition, as summarized by Virchow's triad, that induces vascular damage, venous stasis or activation of blood coagulation.

Our patient was found to have a $1.8 \mathrm{~cm}$ CST incidentally on a two-dimensional echocardiogram. It is likely that the cause was multifactorial but predominantly due to underlying malignancy in addition to sepsis secondary to bacteremia. Our patient was initially borderline hypotensive on admission, likely due to sepsis, but remained hemodynamically stable throughout the hospital course.

The majority of reported cases of CST are associated with cases involving intra-cardiac instrumentation or endocarditis. Cases have been reported in patients undergoing right heart catheterization, post-surgical repair of Ebstein's anomaly, insertion of central venous catheters and Swan-Ganz catheters, and pacing wires or lead wires of defibrillators [7-10]. Infectious causes, regardless of proximity to the heart, have also been reported with CST [11].

Early recognition and clinical suspicion are critical as this rare complication has life-threatening complications including cardiac tamponade, acute myocardial infarction and cardiac ar- rest [11]. An acute obstruction to the outflow of venous blood from the heart will immediately lead to an increase in the intravascular pressure. With a lack of collateral outflow, increasing pressures ultimately result in vascular compromise via vascular rupture or a buildup of transudative fluid due to elevated hydrostatic pressures [12]. The fluid collection can then result in tamponade and hemodynamic collapse.

Unfortunately, CST is often undiagnosed given the rarity and non-specific presentation of disease. Many reported cases are diagnosed on autopsies. Otherwise, commonly reported symptoms in patients found to have a CST include shortness of breath, hypotension, chest pain and ischemic changes on electrocardiogram $[1,2]$. A CST can be readily identified on transthoracic echocardiography as well as computed tomography imaging of the chest with intravascular contrast. The most suggestive sign, aside from visible clot, is coronary sinus dilation [4].

CST is a life-threatening condition that has not been studied enough to guide management. More research is necessary to establish guidelines on the management and approach to treating CST. As a thrombus, the two general treatment options are naturally anticoagulation or thrombectomy. As there are no established guidelines on therapy, most incidences are treated on a case-by-case basis depending on the availability and experience of specialists. Neri et al described a case of a 79-year-old gentleman that had significant improvement 
of left ventricular function following thrombo-embolectomy [13]. Otherwise, there are very limited published data on the individual management of CST particularly with the use of heparin or direct oral anticoagulants.

\section{Conclusion}

As previously stated, CST is a rare but life-threatening condition associated with any disease that increases the likelihood of thrombus formation. The majority of cases reported in the literature include patients that had cardiac instrumentation prior to clot formation. The vague presentation in addition to potentially rapid progression and hemodynamic involvement highlight the importance of clinical suspicion of this condition. A major hurdle in understanding CST also stems from our lack of understanding of the morphology, flow rates and average dimensions of the sinus itself. The medical community would benefit from more research on the relative size of a coronary sinus in relation to age, body habitus, and other factors that can play a role in the caliber of the vessel itself. This information would allow us to understand at which point a lesion becomes hemodynamically significant based on alterations in flow rates and patterns. CST can likely be managed with anticoagulation or mechanical clot retrieval; however, more documented cases and research are vital in establishing guidelines for effective treatment.

\section{Acknowledgments}

None to declare.

\section{Financial Disclosure}

None to declare.

\section{Conflict of Interest}

None to declare.

\section{Informed Consent}

Informed consent was obtained from the patient.

\section{Author Contributions}

This case report was a joint effort by Anton Mararenko, Firas Ajam, Abbas Alshami, and David Zagha. The case was reviewed and discussed as a team. All involved contributed equally to this project.

\section{Data Availability}

The authors declare that data supporting the findings of this study are available within the article.

\section{References}

1. Parmar RC, Kulkarni S, Nayar S, Shivaraman A. Coronary sinus thrombosis. J Postgrad Med. 2002;48(4):312313.

2. Kachalia A, Sideras P, Javaid M, Muralidharan S, Stevens-Cohen P. Extreme clinical presentations of venous stasis: coronary sinus thrombosis. J Assoc Physicians India. 2013;61(11):841-843.

3. Yeo KK, Davenport J, Raff G, Laird JR. Life-threatening coronary sinus thrombosis following catheter ablation: case report and review of literature. Cardiovasc Revasc Med. 2010;11(4):262 e261-265.

4. Frogel JK, Weiss SJ, Kohl BA. Transesophageal echocardiography diagnosis of coronary sinus thrombosis. Anesth Analg. 2009;108(2):441-442.

5. Blann AD. How a damaged blood vessel wall contibutes to thrombosis and hypertenasion. Pathophysiol Haemost Thromb. 2003;33(5-6):445-448.

6. George F, Brouqui P, Boffa MC, Mutin M, Drancourt M, Brisson C, Raoult D, et al. Demonstration of Rickettsia conorii-induced endothelial injury in vivo by measuring circulating endothelial cells, thrombomodulin, and von Willebrand factor in patients with Mediterranean spotted fever. Blood. 1993;82(7):2109-2116.

7. Milligan G, Moscona JC. Coronary sinus thrombosis: Echocardiographic visualization in a patient with known risk factors. J Clin Ultrasound. 2018;46(8):555-557.

8. Shimbori R, Takaki J, Hosoda Y, Hidaka H, Okamoto K, Fukui T. Enlarged coronary sinus thrombosis after repair of Ebstein's anomaly. J Card Surg. 2020;35(3):675-678.

9. O'Cochlain B, Delurgio D, Leon A. Biventricular pacing using two pacemakers and the triggered VVT mode. Pacing Clin Electrophysiol. 2001;24(8 Pt 1):1284-1285.

10. Suarez-Penaranda JM, Rico-Boquete R, Munoz JI, Rodriguez-Nunez A, Martinez Soto MI, Rodriguez-Calvo M, Concheiro-Carro L. Unexpected sudden death from coronary sinus thrombosis. An unusual complication of central venous catheterization. J Forensic Sci. 2000;45(4):920922.

11. Hart MA, Simegn MA. Pylephlebitis presenting as spontaneous coronary sinus thrombosis: a case report. J Med Case Rep. 2017;11(1):309.

12. Stewart RH, Rohn DA, Allen SJ, Laine GA. Basic determinants of epicardial transudation. Am J Physiol. 1997;273(3 Pt 2):H1408-1414.

13. Neri E, Tripodi A, Tucci E, Capannini G, Sassi C. Dramatic improvement of LV function after coronary sinus thromboembolectomy. Ann Thorac Surg. 2000;70(3):961963. 